\title{
Circadian-Based Therapies for Circadian Rhythm Sleep-Wake Disorders
}

\author{
Helen J. Burgess $^{1}$ • Jonathan S. Emens ${ }^{2}$
}

Published online: 2 July 2016

(C) Springer International Publishing AG 2016

\begin{abstract}
This review summarizes recent developments relevant to the treatment of circadian rhythm sleepwake disorders. The clinical practice guidelines for the treatment of intrinsic circadian rhythm sleep-wake disorders are described, followed by recent treatment studies for delayed sleep-wake phase disorder, non-24-hour sleep-wake disorder, irregular sleep-wake disorder, and shift work. New methods to estimate circadian phase, including home saliva collection to estimate the dim light melatonin onset, circadian questionnaires, and general rules to guide light and exogenous melatonin treatments, are described. New developments in light treatment are detailed, including light flashes during sleep and wearable light devices. Substances such as caffeine and alcohol, and devices such as electronic tablets are also considered for their potential to shift circadian timing. Finally, an update on melatonin supplements in the USA is discussed, along with the controversy
\end{abstract}

This article is part of the Topical Collection on Behavioral Therapy

Helen J. Burgess

Helen_J_Burgess@rush.edu

Jonathan S. Emens

emensj@ohsu.edu

1 Biological Rhythms Research Laboratory, Department of Behavioral Sciences, Rush University Medical Center, 1645 West Jackson Blvd., Suite 425, Chicago, IL 60612, USA

2 Departments of Psychiatry \& Medicine, Oregon Health and Science University, Portland VA Medical Center, 3710 SW US Veterans Hospital, Rd. P3-PULM, Portland, OR 97239, USA surrounding the use of melatonin supplements in patients with prediabetes or diabetes.

Keywords Advance $\cdot$ Agonist $\cdot$ Alcohol $\cdot$ Caffeine $\cdot$ Diabetes $\cdot$ Delay $\cdot$ Light $\cdot$ Melatonin

\section{Introduction}

This article reviews interesting and important findings published since 2013 relevant to the treatment of circadian rhythm sleep-wake disorders. The recent update to the clinical practice guideline for the treatment of intrinsic circadian rhythm sleepwake disorders is first summarized, followed by a review of recent treatment studies published since then. These include studies assessing treatments for delayed sleep-wake phase disorder, non-24-hour sleep-wake disorder, irregular sleep-wake disorder, and shift work. New approaches to estimate circadian timing are then reviewed, including home saliva collection to directly measure the dim light melatonin onset, and the use of circadian questionnaires to estimate the dim light melatonin onset. General rules to guide light and exogenous melatonin treatments are also presented. New developments in light treatment are also discussed, including light flashes during sleep and wearable light devices. Substances such as caffeine and alcohol, and devices such as electronic tablets are also considered, for their potential to inadvertently shift circadian timing in an unwanted direction. Lastly, an update on melatonin supplements in the USA is discussed, along with the controversy surrounding the use of melatonin supplements in patients with prediabetes or diabetes. 


\section{Update to Clinical Practice Guideline for the Treatment of Intrinsic Circadian Rhythm Sleep-Wake Disorders}

In 2012, the American Academy of Sleep Medicine (AASM) formed a task force to update the practice parameters for the intrinsic circadian rhythm sleep-wake disorders (CRSWDs). Search terms were agreed upon, and a literature search was conducted of relevant articles published since the last practice parameters were issued in 2007. The task force then developed PICO (patient, population, or problem; intervention; comparison; and outcomes; see Table 1 in $[1 \bullet \cdot]$ ) questions that were used in the assessment of the literature. As part of this process, the critical outcomes and the degree of change considered clinically relevant were defined. The task force then chose articles from the literature search for inclusion, and the relevant data were extracted (including study methodology and type, subject population, intervention(s), relevant outcome measures, harms, and limitations). The extracted data were classified by CRSWD, outcome measure, PICO question addressed, study design, and assessment method. Metaanalyses were conducted when possible. Finally, the GRADE (Grading of Recommendations Assessment, Development, and Evaluation) system was used to evaluate the data and arrive at treatment recommendations by taking into account the quality of the evidence, an assessment of risks and benefits, and patient values and preferences.

The use of the more rigorous GRADE system resulted in truly novel CRSWD practice guidelines that were notable for a lack of any recommendation for the majority of disorders and interventions assessed. The recommendations that were made were further notable for the general weakness of recommendation (see Table 5 in $[1 \bullet \bullet$ ). For those interventions without a recommendation, an accompanying editorial made clear that the task force was not indicating that these interventions should not be used by clinicians [2]; rather, the available evidence was insufficient to make a recommendation for or against such interventions. In brief, the practice parameters issued a weak recommendation for the use of light therapy in advanced sleep-wake phase disorder (ASWPD) and irregular sleep-wake rhythm disorder (ISWRD), and a strong recommendation against the use of sleep-promoting medications in ISWRD. There was also a weak recommendation for timed melatonin or melatonin agonists in delayed sleep-wake phase disorder (DSWPD), non-24-hour sleep-wake rhythm disorder (N24SWD), and ISWRD in children/adolescents with neurological disorders, but a weak recommendation against melatonin in elderly demented populations. In terms of combination treatments, there was a weak recommendation for the use of light and behavioral interventions in children/adolescents with DSWPD, and a weak recommendation against the use of melatonin and light in elderly demented individuals with ISWRD. A pictorial summary of the recommendations for treatment is shown in Fig. 1. The general weakness of these practice parameters reflects the deficits in the evidence base and highlights the need for clinical trials for CRSWD interventions [2].

Following the publication of the AASM practice parameters, we published a practical review aimed at helping clinicians treat patients with a CRSWD or those suspected of having some degree of circadian misalignment that impacted disease signs and/or symptoms [3]. Updates to the practice guidelines for the treatment of extrinsic CRSWDs (shift work and jet lag disorders) should be forthcoming from the AASM in the next few years.

\section{More Recent Studies on Circadian Rhythm Sleep-Wake Disorders}

We searched for studies of intrinsic circadian rhythm sleepwake disorders published from 2014 onwards, to identify any studies published after the literature search for the update in clinical practice guideline. We found no new studies of advanced sleep-wake phase disorder. There was one new study of delayed sleep-wake phase disorder. In this study, Saxvig et al. [4] randomized 40 adolescents diagnosed with DSWPD to one of four 2-week treatments: dim morning light plus placebo capsules, bright morning light plus placebo capsules, dim morning light plus melatonin capsules, and bright morning light plus melatonin capsules. All the treatments included the gradual advancement in wake times and capsule and light administration times by $1 \mathrm{~h}$ per day (such prescribed sleepwake scheduling would have classified even the light- or melatonin-only interventions as combination treatments in the AASM CRSWD practice guidelines). Light ("approximately 10,000 lx" versus $400 \mathrm{~lx}$ of "dim red" light) was administered for 30-45 min upon awakening while capsules ( $3 \mathrm{mg}$ melatonin or placebo) were administered $12 \mathrm{~h}$ after waking (but not before $8 \mathrm{pm}$ ). Outcomes included sleep diaries, wrist actigraphy, and salivary dim light melatonin onset (DLMO). No differences were found across the four groups with an approximate $2 \mathrm{~h}$ advance seen in the DLMO and rise time and a $1 \mathrm{~h}$ advance in bedtime. Therefore, none of the interventions either alone or in combination were any more effective than the advancement of wake times alone. The study also included a subsequent 3-month open label trial of melatonin and bright light versus no intervention and found that only the former maintained the shift in sleep to an earlier time.

There were two new publications concerning blind individuals with N24SWD. Most important was Lockley et al.'s study [5•] of the drug tasimelteon in this population which became the first medication approved by the FDA for the treatment of any CRSWD. The study was a randomized, placebo controlled study in 84 individuals with N24SWD 


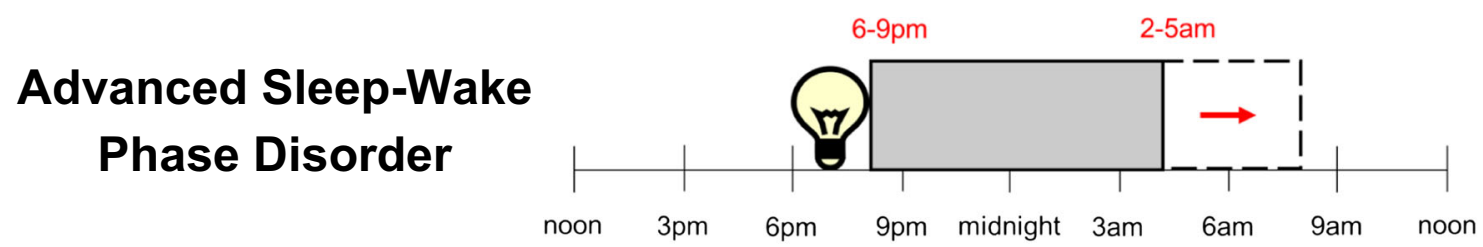

Delayed Sleep-Wake

Phase Disorder

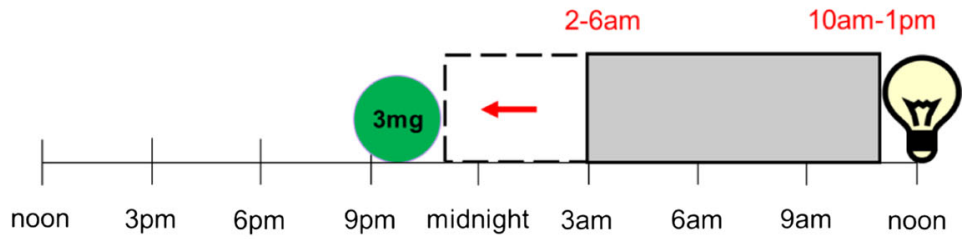

Non-24 Hour

Sleep-Wake Disorder

(Blind, period >24h)

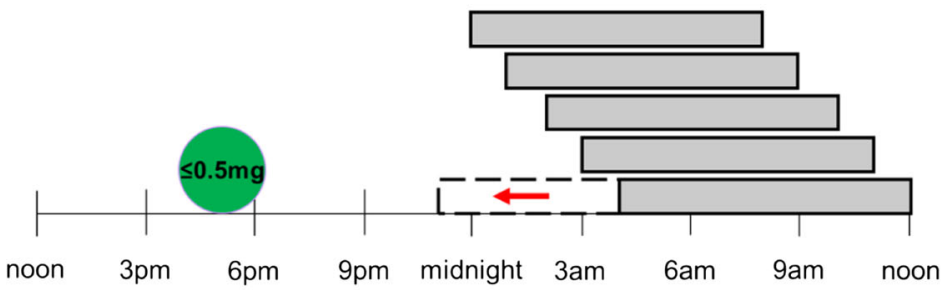

Non-24 Hour

Sleep-Wake Disorder

(Blind, period <24h)

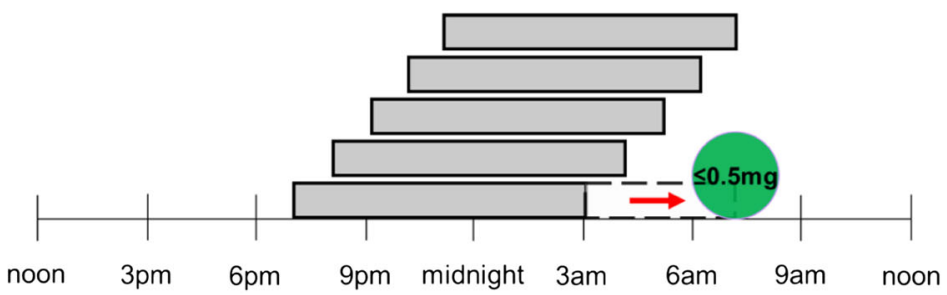

Irregular Sleep-Wake

Disorder-Elderly

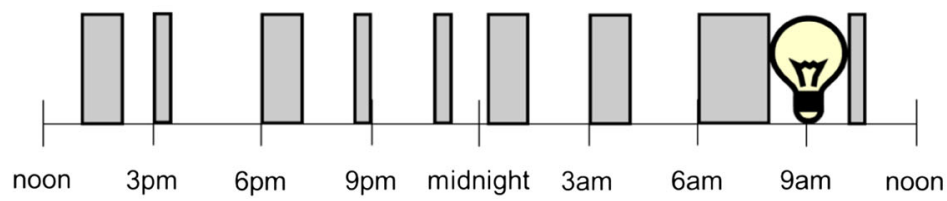

\section{Irregular Sleep-Wake \\ Disorder-Children}

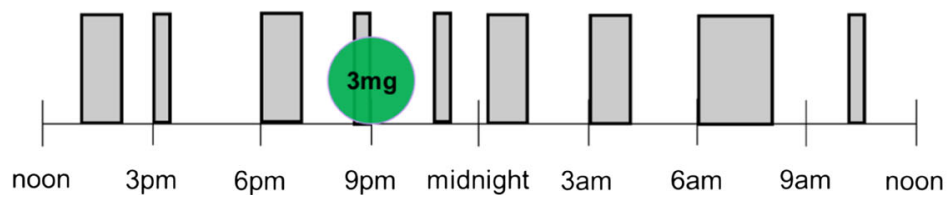

Fig. 1 A pictorial representation of the most recent clinical practice guideline recommendations for treatment of intrinsic circadian rhythm sleep-wake disorders. From top down is advanced sleep-wake phase disorder, delayed sleep-wake phase disorder, non-24-hour sleep-wake disorder in the blind with circadian period $>24$ h, non-24-hour sleepwake disorder in the blind with circadian period $<24 \mathrm{~h}$, irregular sleepwake disorder for the elderly, and irregular sleep-wake disorder for

children. The times in red indicate the range in typical fall asleep or wake times seen in patients prior to treatment, and the red arrow represents the direction of desired phase shifts. The light bulb symbol represents light therapy. The circles represent melatonin pills with doses suggested by the authors to either minimize or maximize melatonin's soporific effect as needed 
comparing $20 \mathrm{mg}$ of tasimelteon and placebo administered $1 \mathrm{~h}$ before a target bedtime. Tasimelteon was found to safely and effectively treat individuals with N24SWD: entrainment (synchronization to the 24-h day) occurred in $20 \%$ ( 8 of 40) of patients receiving the drug compared with $3 \%$ (1 of 38 ) receiving placebo. As noted in the AASM CRSWD practice guideline $[1 \bullet \bullet$, the entrainment rate was lower than the $67 \%$ entrainment rate (12 of 18 patients) found in the metaanalysis of melatonin treatment in N24SWD. However, as noted by the authors themselves, this may have been because entrainment was assessed after just 1 month of treatment. Such a theory is borne out by the higher rates of entrainment found during the longer, open label treatment with tasimelteon. The tasimelteon study also demonstrated improvements (increases and decreases, respectively) in nighttime and daytime sleep (as measured by the worst quartiles of treatment days compared to baseline). Of note, the tasimelteon study could also be considered to include a component of prescribed sleep/wake scheduling as subjects were instructed to maintain selfselected 9-h sleep opportunities with bedtimes within a 4-h range (21:00 to 01:00). The second new publication in N24SWD was a small placebo controlled study by St. Hilaire and Lockley [6] looking at the effects of caffeine on entrainment in three blind individuals with the disorder. Caffeine $(150 \mathrm{mg}$ at $10 \mathrm{am})$ failed to entrain the subjects although it did improve daytime alertness in the setting of circadian misalignment.

A single new study of light therapy in elderly patients with dementia and irregular sleep-wake rhythm disorder (ISWRD) was published by Figueiro et al. [7]. The study looked at 4 weeks of "bluish-white" light in 14 individuals with dementia living in a skilled nursing facility using custom built "luminaires" in the patient's rooms (on from approximately 6-8 am to approximately $6 \mathrm{pm}$ ). Light and activity were measured with a Daysimeter device placed on the wrist, and improvements with light therapy were seen in sleep efficiency and total sleep time as well as an increase in "phasor magnitude" (a quantification of the relationship between the timing and amplitude of the rest-activity rhythm and light-dark timing) that the authors suggested represented an increase in "circadian entrainment." As noted above, AASM practice parameters included a weak recommendation for light therapy in this particular patient population with ISWRD, and the results of this study are consistent with this recommendation.

We also searched for recent studies of shift work and jet lag from 2013 onwards, deemed relevant to treatment. One study reported on the effects of aligning work schedules to individual shift worker's chronotype and found significant improvements in the workers' sleep [8]. Specifically, in an industrial factory, over a 5-month period, Vetter and colleagues abolished morning shifts for late chronotypes and night shifts for early chronotypes, while intermediate chronotypes continued to work morning, evening, and night shifts in a rotating forward shift schedule. In response, they observed in the late and early chronotypes, a significant increase in self-reported sleep duration on work days (24-28 $\mathrm{min}$ ) along with improvements in sleep quality and wellbeing. This study highlights the importance of considering chronotype when assigning work schedules for shift workers.

\section{New Approaches for Measuring Circadian Timing in Patients}

The most reliable marker of circadian timing in humans is the dim light melatonin onset (DLMO) [9], which is the time when endogenous melatonin levels begin to rise in dim light, on average about $2-3 \mathrm{~h}$ before habitual bedtime $[10,11]$. Melatonin must be measured in dim light because light can suppress melatonin secretion [12]. As highlighted by others, the measurement of the DLMO in patients can assist both with the diagnosis of most circadian rhythm sleep-wake disorders, and also with optimizing the timing of bright light and/or exogenous melatonin treatment $[13,14]$. However, the DLMO is not yet commonly measured in the clinic. As a proof of concept study, we recently tested a home kit and procedure with objective measures of compliance to the requirement for dim light levels and scheduled times for saliva samples. In 32 healthy controls, we found that the home DLMOs compared favorably to the standard laboratory-based DLMOs $(r=0.91$, $p<0.001$ ), and only $8 \%$ of home DLMOs were confounded by light or sampling errors [15•]. In a second study of 32 patients with DSWPD, we also found that the home DLMOs compared favorably to the standard laboratory-based DLMOs $(r=0.93, p<0.001)$ [16 ]. Compliance to the home procedures was also good in these patients, but light and sampling errors occurred more frequently around the time of the DLMO, resulting in greater data loss $(17 \%$ of the home DLMOs were invalid) [16•]. Overall, these studies demonstrate that accurate DLMOs can be assessed in the home, and we hope that in the future home-based assessment of the DLMO for the diagnosis and treatment of CRSWD becomes the norm [17].

However, when measuring the DLMO is not feasible, one approach to estimating circadian phase could be to use circadian questionnaires that ask people about the timing of their behavior, such as their sleep. Two commonly used questionnaires include the Morningness-Eveningness Questionnaire (MEQ) [18] from which a MEQ chronotype score can be derived and the Munich ChronoType Questionnaire [19] from which a midpoint of sleep on work-free days, sleep corrected (MSFsc) chronotype value can be derived. In 2014, Kitamura and colleagues [20] reported results from the first study to assess the MEQ score, MSFsc, and DLMO in the same sample of subjects $(n=44)$. They found the DLMO was significantly correlated with the MEQ score $(r=-0.40, p=0.055)$ and 
$\operatorname{MSFsc}(r=0.54, p<0.001)$. Recently, in a larger sample $(n=$ 60 ) with a larger range in chronotype and DLMO, we found stronger correlations between DLMO, MEQ score, and MSFsc [21•]. In this case, both MEQ score and MSFsc were essentially similarly correlated with the DLMO (MEQ score $r=-0.70, p<0.001$; MSFsc $r=0.68, p<0.001)$. However, we noted a range of about $4 \mathrm{~h}$ in the DLMO around each chronotype score, suggesting these measures of chronotype are too variable to be used to time circadian-based treatments, as this could result in the mistiming of treatments relative to the DLMO, potentially worsening the circadian misalignment [21 $]$. It remains to be determined how closely phase shifts in the DLMO can be reflected in changes in these chronotype scores.

Currently, instead of estimating the DLMO from chronotype scores, we use rough rules of thumb to guide light and melatonin treatment when measuring the DLMO is not feasible [3, 22]. For example, for phase advancing light treatments, we tend to avoid starting the light treatment more than $1 \mathrm{~h}$ earlier than spontaneous wake time to avoid accidental phase delays. Phase delaying light can extend into the first $3 \mathrm{~h}$ or so of the habitual sleep episode. For melatonin treatments, we tend to estimate the DLMO about 2-3 h before bedtime, such that melatonin is taken about $5 \mathrm{~h}$ before habitual bedtime to phase advance and around habitual wake time to phase delay. We typically instruct patients to take low doses $(\leq 0.5 \mathrm{mg})$ when they need to minimize the soporific effect of exogenous melatonin, and high doses $(3 \mathrm{mg})$ when falling asleep (a dark pulse) would assist in the desired phase shift (Fig. 1). Ultimately, however, the treatment of CRSWD will be optimized once the direct assessment of the DLMO in the clinic or in people's homes becomes more commonly available.

\section{New Methods of Light Administration}

The main burden typically associated with light treatment is the time commitment, such as a $30-\mathrm{min}$ to $1-\mathrm{h}$ treatment per day, and/or the usual requirement to be seated close to a light box in order to receive light of sufficient intensity [3]. To avoid the daily time commitment during wakefulness, there has been much interest in applying light treatment during sleep, when the circadian system is usually very sensitive to light. Recently, a 1-h interval of millisecond flashes of bright light from a flash bulb placed above people's heads while they slept was found to significantly phase delay the DLMO by about $30 \mathrm{~min}[23 \bullet]$. The $1-\mathrm{h}$ interval of light flashes was timed to occur during the third hour of the habitual sleep episode. The bright light was of sufficient intensity to still reach the retina after passing through closed eyelids, without major disruption to sleep [23•]. Indeed, such light flashes may sometimes produce larger phase delays than seen with continuous light [24]. Others have recently reported on a light mask worn during sleep that can also phase delay circadian timing, up to 30 min after a 60-min light exposure window during the first $2 \mathrm{~h}$ of the habitual sleep episode [25]. While these recent findings are promising, to our knowledge, no such light at night systems or light masks are currently commercially available.

The requirement to be seated close to a light box for $30 \mathrm{~min}$ to $1 \mathrm{~h}$ per day likely contributes to adherence issues in home treatments. Two wearable light devices are now available commercially. The Luminette 2 device (Myluminette.com) resembles a visor, with the light source positioned above the eyes. The Re-timer device (Re-timer.com) is more similar to glasses, and the light source is positioned below the eyes. Both devices are lightweight, very portable, and easily charged; have good battery life; have adjustable light intensity to accommodate patient preference; and importantly permit the patient to be ambulatory during light treatment. In our opinion, these wearable devices are likely to improve compliance to light treatment. While there is evidence that an earlier prototype of the Re-timer could shift circadian timing [26], we await publications that conclusively demonstrate that these devices can shift circadian timing.

\section{Substances and Devices That May Alter Circadian Timing}

As noted above, caffeine failed to result in entrainment in a small placebo controlled study in blind individuals with N24SWD [6] who would have required daily phase advances to effectively treat their disorder (shifts in the timing of the circadian pacemaker to an earlier time). However, another study recently demonstrated that caffeine is capable of causing phase delays in the timing of the circadian pacemaker raising the possibility that caffeine could be used as a true chronobiotic. Burke et al. [27•] administered $2.9 \mathrm{mg} / \mathrm{kg}$ of caffeine $3 \mathrm{~h}$ before habitual bedtime in a double-blind, placebo controlled fashion in five healthy individuals and found that caffeine caused a phase delay that was approximately $40 \mathrm{~min}$ longer than the dim light placebo control. In comparison, the response to a 3-h exposure of $\sim 3000 \mathrm{~lx}$ beginning at habitual bedtime was about $85 \mathrm{~min}$ longer than the dim light placebo. Therefore, caffeine might have potential as a phase delaying agent in ASWPD or in N24SWD patients with circadian periods that are less than $24 \mathrm{~h}$ and therefore require corrective phase delays to achieve entrainment. However, first, it would have to be determined whether the direct alerting effects of caffeine would negate any circadian resetting benefits in these disorders and whether the dose-response and phase-response curves for caffeine are favorable for its use in CRSWDs.

A single dose of alcohol can alter phase shifts to light in rodents, presumably due to the alcohol directly altering levels 
of GABA and glutamate in the suprachiasmatic nuclei, the location of the central circadian pacemaker [28, 29]. We recently conducted two placebo controlled, within subject design studies to determine for the first time if a single dose of alcohol (0.6-0.8 g/kg, approximating 2-3 standard drinks) can alter phase shifts to light in humans [30•]. In one study, 14 healthy subjects consumed alcohol (vs. placebo) in the late evening immediately before a 2 -h bright light treatment, to test the effect of alcohol on phase delays to light. In the other study, ten healthy subjects consumed alcohol (vs. placebo) in the early morning, immediately before a 2 -h bright light treatment, to test the effect of alcohol on phase advances to light. In both studies, there was not a consistent response, with alcohol increasing phase shifts to light in some individuals, but decreasing phase shifts to light in other individuals. Additionally, the differences in phase shifts between alcohol and placebo were on average always less than $30 \mathrm{~min}$, indicating the changes in phase shifts were not meaningful. It remains to be determined if chronic alcohol consumption can alter phase shifts to light.

Finally, the use of light-emitting devices must be considered both as potential interventions in CRSWDs and, more likely, as precipitators or perpetuators of these disorders. Chang et al. [31] demonstrated in a randomized cross-over laboratory study that exposure to a light-emitting electronic book (e-Book) for approximately $4 \mathrm{~h}$ prior to bedtime over five evenings caused a phase delay compared to reading a printed book for the same period (the DLMO occurred $\sim 1.5 \mathrm{~h}$ later in the e-Book condition). This study was criticized as lacking generalizability due to the daytime light levels ( 90 lx); the subjects were exposed to in the laboratory-the contention being that most individuals experience brighter light conditions during their waking day outside the laboratory and that such relatively dim light might have caused increased sensitivity of the circadian system to the resetting effects of the e-Books [32]. However, this study and others [33] highlight the impact of industrial lighting conditions on biological timing. Specifically, they are consistent with the hypothesis that a significant delay in the timing of the biological clock occurs under conditions of artificial light exposure. To what extent the exposure to industrial lighting conditions contributes to the development of some CRSWDs remains to be demonstrated as does the potential impact, in CRSWDs, of countermeasures targeting this same exposure [34].

\section{Update on Exogenous Melatonin}

The development of several melatonin agonists (including tasimelteon noted above) has brought to the fore the question of whether exogenous melatonin itself or melatonin agonists are most appropriate for the treatment of CRSWDs. Melatonin was given a weak recommendation for the treatment of
DSWPD, N24SWD, and children and adolescents with neurological disorders and ISWRD in the AASM practice parameters. As we have noted previously, melatonin is classified as a dietary supplement by the FDA and therefore is not regulated like pharmaceuticals. This has led to questions regarding both the purity and the dose accuracy of various melatonin preparations. However, choosing a melatonin preparation from a supplier that participates in the USP verified program for dietary supplements might address these issues. Indeed, in 2015, a melatonin USP-certified product was added to the national formulary of the Veterans Health Administration (http:/www. pbm.va.gov/clinicalguidance/drugmonographs.asp) which is the largest integrated health care system in the USA (www. va.gov/health). The specific indications included DSWPD and N24SWD.

The potential contraindications to melatonin use have included excessive sedation with ingestion, pregnancy or nursing, seizure disorders, and warfarin therapy. More recently, the question of the potential effects of exogenous melatonin on glucose tolerance has been raised despite the fact that lower endogenous levels of melatonin have been found to be associated with increased risk of type II diabetes [35]. RubioSastre et al. demonstrated that $5 \mathrm{mg}$ of exogenous melatonin acutely impaired glucose tolerance [36]. The same group has recently also showed that a common genetic variant that is associated with increased type II diabetes risk is also associated with worse glucose tolerance in response to exogenous melatonin [37]. As the potential metabolic consequences of melatonin administration become clearer, they will have to be weighed against the possible metabolic consequences of circadian misalignment associated with the CRSWDs themselves [38, 39].

\section{Conclusions}

This review provided an update on recent findings and developments that are relevant to the treatment of CRSWD. These new findings ranged from the new update to the clinical practice guideline for the treatment of intrinsic CRSWD, to recent treatment studies published since then. New approaches to estimate circadian timing, new developments in light treatment, and consideration of substances and devices that may inadvertently shift circadian timing were also reviewed. Lastly, recent developments regarding melatonin supplements were discussed. It is clear that large randomized clinical trials testing combination treatments in patients with carefully defined CRSWD are sorely needed [2]. Field-based studies are also required, as laboratory settings often lead to an artificial restriction in activities and movement, that can for example, artificially reduce the high variability in light exposure people often receive in their homes [40]. 
Acknowledgments We thank Muneer Rizvydeen for his assistance in creating the figures. HJB is supported by grants from the National Center for Complementary and Integrative Health, and the National Institute on Alcohol Abuse and Alcoholism. The content is solely the responsibility of the authors and does not necessarily represent the official views of the National Institutes of Health.

\section{Compliance with Ethical Standards}

Conflict of Interest Helen J. Burgess and Jonathan S. Emens declare that they have no conflict of interest.

Human and Animal Rights and Informed Consent This article does not contain any studies with human or animal subjects performed by any of the authors.

\section{References}

Papers of particular interest, published recently, have been highlighted as:

- Of importance

•- Of major importance

1.• Auger RR, Burgess HJ, Emens JS, Deriy LV, Thomas SM, Sharkey KM. Clinical practice guideline for the treatment of intrinsic circadian rhythm sleep-wake disorders: advanced sleep-wake phase disorder (ASWPD), delayed sleep-wake phase disorder (DSWPD), non-24-hour sleep-wake rhythm disorder (N24SWD), and irregular sleep-wake rhythm disorder (ISWRD). An update for 2015: an American Academy of Sleep Medicine Clinical Practice Guideline. J Clin Sleep Med. 2015;11(10):1199-236. Most recent update to clinical practice guidelines for intrinsic circadian rhythm sleep-wake disorders.

2. Auger RR, Burgess HJ, Emens J, Deriy LV, Sharkey KM. Do evidence-based treatments for circadian rhythm sleep-wake disorders make the GRADE? Updated guidelines point to need for more clinical research. J Clin Sleep Med. 2015;11(10):1079-80.

3. Emens J, Burgess HJ. Effect of light and melatonin and other melatonin receptor agonists on human circadian physiology. Sleep Med Clin. 2015;10:435-53.

4. Saxvig IW, Wilhelmsen-Langeland A, Pallesen S, Vedaa O, Nordhus IH, Bjorvatn B. A randomized controlled trial with bright light and melatonin for delayed sleep phase disorder: effects on subjective and objective sleep. Chronobiol Int. 2014;31:72-86.

5. Lockley SW, Dressman MA, Licamele L, Xiao C, Fisher DM, Flynn-Evans EE, et al. Tasimelteon for non-24-hour sleep-wake disorder in totally blind people (SET and RESET): two multicentre, randomised, double-masked, placebo-controlled phase 3 trials. Lancet. 2015;386(10005):1764. First medication approved by the FDA for the treatment of any circadian rhythm sleepwake disorder.

6. St Hilaire MA, Lockley SW. Caffeine does not entrain the circadian clock but improves daytime alertness in blind patients with non-24hour rhythms. Sleep Med. 2015;16(6):800-4.

7. Figueiro MG, Plitnick BA, Lok A, Jones GE, Higgins P, Hornick $\mathrm{TR}$, et al. Tailored lighting intervention improves measures of sleep, depression, and agitation in persons with Alzheimer's disease and related dementia living in long-term care facilities. Clin Interv Aging. 2014;9:1527-37.
8. Vetter C, Fischer D, Matera JL, Roenneberg T. Aligning work and circadian time in shift workers improves sleep and reduces circadian disruption. Curr Biol. 2015;25(7):907-11.

9. Klerman EB, Gershengorn HB, Duffy JF, Kronauer RE. Comparisons of the variability of three markers of the human circadian pacemaker. J Biol Rhythms. 2002;17(2):181-93.

10. Lewy AJ, Cutler NL, Sack RL. The endogenous melatonin profile as a marker of circadian phase position. J Biol Rhythms. 1999;14(3):227-36.

11. Burgess HJ, Fogg LF. Individual differences in the amount and timing of salivary melatonin secretion. PLoS One. 2008;3(8): e3055.

12. Lewy AJ, Wehr TA, Goodwin FK, Newsome DA, Markey SP. Light suppresses melatonin secretion in humans. Science. 1980;210(4475):1267-9.

13. Lockley SW. Timed melatonin treatment for delayed sleep phase syndrome: the importance of knowing circadian phase. Sleep. 2005;28:1214-6.

14. Keijzer H, Smits MG, Duffy JF, Curfs LM. Why the dim light melatonin onset (DLMO) should be measured before treatment of patients with circadian rhythm sleep disorders. Sleep Med Rev. 2014;18(4):333-9.

15. Burgess HJ, Wyatt JK, Park M, Fogg LF. Home circadian phase assessments with measures of compliance yield accurate dim light melatonin onsets. Sleep. 2015;38(6):889-97. Study demonstrating that with appropriate measures of compliance, accurate DLMOs can be collected by people at home.

16. Burgess HJ, Park M, Wyatt J, Fogg LF. Home dim light melatonin onsets with measures of compliance in delayed sleep phase disorder. J Sleep Res. in press. Study demonstrating that with appropriate measures of compliance, accurate DLMOs can be collected by patients with delayed sleep wake phase disorder at home.

17. Rahman SA, Kayumov L, Tchmoutina EA, Shapiro CM. Clinical efficacy of dim light melatonin onset testing in diagnosing delayed sleep phase syndrome. Sleep Med. 2009;10(5):549-55.

18. Horne JA, Ostberg O. A self-assessment questionnaire to determine morningness-eveningness in human circadian rhythms. Int $\mathrm{J}$ Chronobiol. 1976;4:97-110.

19. Roenneberg T, Wirz-Justice A, Merrow M. Life between clocks: daily temporal patterns of human chronotypes. J Biol Rhythms. 2003;18(1):80-90.

20. Kitamura S, Hida A, Aritake S, Higuchi S, Enomoto M, Kato M, et al. Validity of the Japanese version of the Munich ChronoType Questionnaire. Chronobiol Int. 2014;31(7):845-50.

21. Kantermann T, Sung H, Burgess HJ. Comparing the morningnesseveningness questionnaire and Munich ChronoType Questionnaire to the dim light melatonin onset. J Biol Rhythms. 2015;30(5):44953. A comparison of circadian questionnaires with the dim light melatonin onset in the largest sample to date.

22. Eastman CI, Burgess HJ. How to travel the world without jet lag. Sleep Med Clin. 2009;4:241-55.

23. Zeitzer JM, Fisicaro RA, Ruby NF, Heller HC. Millisecond flashes of light phase delay the human circadian clock during sleep. J Biol Rhythms. 2014;29(5):370-6. Demonstration that brief flashes of light above the bed can shift circadian timing during sleep without major disruption to sleep.

24. Najjar RP, Zeitzer JM. Temporal integration of light flashes by the human circadian system. J Clin Invest. 2016;126(3):938-47.

25. Figueiro MG, Bierman A, Rea MS. A train of blue light pulses delivered through closed eyelids suppresses melatonin and phase shifts the human circadian system. Nat Sci Sleep. 2013;5:133-41.

26. Wright HR, Lack LC, Partridge KJ. Light emitting diodes can be used to phase delay the melatonin rhythm. J Pineal Res. 2001;31: $350-5$.

27. Burke TM, Markwald RR, McHill AW, Chinoy ED, Snider JA, Bessman SC, et al. Effects of caffeine on the human circadian clock 
in vivo and in vitro. Sci Transl Med. 2015;7(305):305ra146. Demonstration that caffeine can phase delay circadian timing.

28. Ruby CL, Prosser RA, DePaul MA, Roberts RJ, Glass JD. Acute ethanol impairs photic and nonphotic circadian phase resetting in the Syrian hamster. Am J Physiol. 2009;296(2): R411-8.

29. Brager AJ, Ruby CL, Prosser RA, Glass JD. Acute ethanol disrupts photic and serotonergic circadian clock phase-resetting in the mouse. Alcohol Clin Exp Res. 2011;35(8):1467-74.

30. Burgess HJ, Rizvydeen M, Fogg LF, Keshavarzian A. A single dose of alcohol does not meaningfully alter circadian phase advances and phase delays to light in humans. Am J Physiol. 2016;310(8):R75965. First study of whether alcohol can alter circadian phase shifts to light in humans.

31. Chang AM, Aeschbach D, Duffy JF, Czeisler CA. Evening use of light-emitting eReaders negatively affects sleep, circadian timing, and next-morning alertness. Proc Natl Acad Sci U S A. 2015;112(4):1232-7.

32. Zeitzer JM. Real life trumps laboratory in matters of public health. Proc Natl Acad Sci U S A. 2015;112(13):E1513.

33. Wright Jr KP, McHill AW, Birks BR, Griffin BR, Rusterholz T, Chinoy ED. Entrainment of the human circadian clock to the natural light-dark cycle. Curr Biol. 2013;23(16):1554-8.
34. van der Lely S, Frey S, Garbazza C, Wirz-Justice A, Jenni OG, Steiner R, et al. Blue blocker glasses as a countermeasure for alerting effects of evening light-emitting diode screen exposure in male teenagers. J Adolesc Health. 2015;56(1):113-9.

35. McMullan CJ, Schernhammer ES, Rimm EB, Hu FB, Forman JP. Melatonin secretion and the incidence of type 2 diabetes. JAMA. 2013;309(13):1388-96.

36. Rubio-Sastre P, Scheer FA, Gomez-Abellan P, Madrid JA, Garaulet $\mathrm{M}$. Acute melatonin administration in humans impairs glucose tolerance in both the morning and evening. Sleep. 2014;37(10):1715-9.

37. Garaulet M, Gomez-Abellan P, Rubio-Sastre P, Madrid JA, Saxena $\mathrm{R}$, Scheer FA. Common type 2 diabetes risk variant in MTNR1B worsens the deleterious effect of melatonin on glucose tolerance in humans. Metabolism. 2015;64(12):1650-7.

38. Wong PM, Hasler BP, Kamarck TW, Muldoon MF, Manuck SB. Social jetlag, chronotype, and cardiometabolic risk. J Clin Endocrinol Metab. 2015;100(12):4612-20.

39. Scheer FA, Hilton MF, Mantzoros CS, Shea SA. Adverse metabolic and cardiovascular consequences of circadian misalignment. Proc Natl Acad Sci U S A. 2009;106(11):4453-8.

40. Crowley SJ, Molina TA, Burgess HJ. A week in the life of full-time office workers: work day and weekend light exposure in summer and winter. Appl Ergon. 2015;46 Pt A:193-200. 